This note was published in IEEE Transactions on Automatic Control, Vol 58 (2), pp. 459-465, 2013, doi: 10.1109/TAC.2012.2206721 and is available at http://ieeexplore.ieee.org/stamp/stamp.jsp?tp=\&arnumber=6228502

(C) 2013 IEEE. Personal use of this material is permitted. Permission from IEEE must be obtained for all other uses, in any current or future media, including reprinting/republishing this material for advertising or promotional purposes, creating new collective works, for resale or redistribution to servers or lists, or reuse of any copyrighted component of this work in other works. 


\title{
LMI-based Stability Criteria for Discrete-time Lur'e Systems with Monotonic, Sector- and Slope-restricted Nonlinearities
}

\author{
N. Syazreen Ahmad, W.P. Heath and Guang Li
}

\begin{abstract}
This note presents new LMI-based stability criteria for the discrete-time multivariable Lur'e system with nonlinearities that are monotonic, sector- and slope-restricted. Corresponding Lur'e-Lyapunov functions are constructed for such a system. The new criteria are expressed in a reasonably general form that can be applied to both non-diagonal and diagonal nonlinearities. We explicitly compare the new approach to the existing LMIbased Popov-like criteria in the literature, and express the results in terms of an Integral Quadratic Constraint (IQC). The applications of the new criteria to some control problems and strategies are briefly discussed. Numerical examples are included to show their performance, and they are shown to be less conservative than the previous results.
\end{abstract}

Notation: We write $x_{k}$ for $x(k), x_{k}^{i}$ for $x(k)$ at coordinate $i$, $V_{k}$ for $V\left(\xi_{k}\right)$ where $\xi_{k}$ is the variable of the function $V$, and $\left(x_{1}, x_{2}\right)$ for a vector representing $\left[x_{1}^{T}, \quad x_{2}^{T}\right]^{T}$. If $M \in \mathbb{C}^{r \times r}$, then $\operatorname{Re}(M)$ is the real value of $M$, and $\mathrm{He}(M)=M+M^{*}$. If $N \in \mathbb{R}^{r \times r}$ is positive semi-definite, then $N^{\frac{1}{2}}$ is the positive semi-definite square root of $N$.

\section{INTRODUCTION}

The discrete-time Lur'e system considers a stable, strictly proper linear time-invariant system

$x_{k+1}=A x_{k}+B u_{k} ; \quad y_{k}=C x_{k}$

which is asymptotically stable with $x_{k} \in \mathbb{R}^{n x} ; u_{k}, y_{k} \in \mathbb{R}^{p}$. Thus $A \in \mathbb{R}^{n_{x} \times n_{x}}, B \in \mathbb{R}^{n_{x} \times p}$ and $C \in \mathbb{R}^{p \times n_{x}}$. The transfer function of the system is given by

$G(z)=C(z I-A)^{-1} B$.

The system is in negative feedback with a memoryless, timeinvariant (static) nonlinearity $\phi: \mathbb{R}^{p} \rightarrow \mathbb{R}^{p}$ that is locally Lipschitz in $y_{k}$ with the relation

$u_{k}=-\phi\left(y_{k}\right)$.

In the early 1960's, many discrete-time Popov-like criteria were developed for the SISO case via the frequency domain approach [24], [26], [25], [9], [10], [23]. Unlike the continuous-time case, the discrete-time counterparts of the Popov criterion have several versions depending on the properties of the nonlinearities. To distinguish the existing results,

N. Syazreen Ahmad and W.P. Heath are with the Control Systems Centre, School of Electrical and Electronic Engineering, University of Manchester, Sackville Street Building, Manchester M13 9PL, UK. (e-mail:Nursyazreen.Ahmad@postgrad.manchester.ac.uk; william.heath@manchester.ac.uk)

G. Li is with the School of Engineering, Computing and Mathematics, Harrison Building, Streatham Campus, University of Exeter, North Park Road, Exeter, UK, EX4 4QF (e-mail:G.Li@exeter.ac.uk)

*Financial support from the Universiti Sains Malaysia (USM) is gratefully acknowledged. consider first the nonlinearity $\phi\left(y_{k}\right)$ which satisfies $\phi(0)=0$, and also the following conditions:

$$
\begin{aligned}
& \phi(y)^{T}\left(K^{-1} \phi(y)-y\right) \leq 0 \quad \forall y \\
& {[\phi(y)-\phi(\hat{y})]^{T}\left[S^{-1}[\phi(y)-\phi(\hat{y})]-(y-\hat{y})\right] \leq 0 \quad \forall \hat{y} \neq y}
\end{aligned}
$$

where $K$ and $S$ are positive definite matrices. The inequality in (4) corresponds to the sector bound conditions, whereas the inequality (5) corresponds to the slope restrictions of the nonlinearities. In the SISO case, the conditions (4) and (5) reduce to

$0 \leq \frac{\phi(y)}{y} \leq K \quad \forall y \quad$ and $\quad 0 \leq \frac{\phi(y)-\phi(\hat{y})}{y-\hat{y}} \leq S \quad \forall \hat{y} \neq y$

respectively. All the nonlinearities belonging to the above conditions will be denoted by $\Omega(K, S)$. The lower slope bound of zero means that the nonlinearity is nondecreasing or monotonic. In a case where the lower slope bound of the nonlinearity is $-S$, the corresponding set will then be denoted by $\hat{\Omega}(K, S)$, and it follows directly that $\Omega(K, S) \subseteq \hat{\Omega}(K, S)$.

Tsypkin (1962) [24] was among the first to propose the frequency-domain criterion which is analogous to the circle criterion in discrete-time domain. As for the discrete-time counterparts of Popov criterion, there are two main results published in 1964, namely the Tsypkin [25] and Jury-Lee [9], [10] criteria. For the SISO case, Soliman and Kwoh (1969) [22] proposed combining the two criteria, but this idea does not seem to have been pursued in the literature until recently. Both the Tsypkin and Jury-Lee criteria were originally developed via the frequency-domain approach for the SISO case. The Tsypkin criterion [25] says that, for the nonlinearity belonging to $\Omega(K, \infty)$, the SISO system is absolutely stable if $\operatorname{Re}\left[K^{-1}+\left(1+\left(1-z^{-1}\right) N\right) G(z)\right]>0$ for all $|z|=1$ is satisfied with $N \geq 0$. Kapila and Haddad [12] extend the Tsypkin criterion to the diagonal MIMO case and prove it via a Lyapunov function of the form

$V_{k}^{1}=\zeta_{k}^{T} P \zeta_{k}+2 \sum_{i=1}^{p} n_{i} \int_{0}^{y_{k}^{i}} \phi_{i}(\sigma) d \sigma$

with $\zeta_{k}=\left(x_{k}, y_{k}\right), P>0, K=\operatorname{diag}\left(k_{1}, \ldots, k_{p}\right)>0$ and $N=\operatorname{diag}\left(n^{1}, \ldots, n^{p}\right) \geq 0$. The conservatism of this approach is further reduced in Park and Kim (1998) [17] by extending the Tsypkin frequency condition to

$\mathrm{He}\left[K^{-1}+\left(I+\left(1-z^{-1}\right) N+(1-z) M\right) G(z)\right]>0$

$\forall|z|=1$, where $M=\operatorname{diag}\left(m_{1}, \ldots, m_{p}\right)$ is an additional positive semi-definite multiplier with $N M=0$. The corresponding Lyapunov function for this case is given by

$V_{k}^{2}=V_{k}^{1}+2 \sum_{i=1}^{p} m_{i} \int_{0}^{y_{k+1}^{i}}\left[k_{i} \sigma-\phi_{i}(\sigma)\right] d \sigma$. 
As for the Jury-Lee criteria [10], [11], the MIMO system with nonlinearities that belong to $\hat{\Omega}(K, S)$ (with $S \geq K$ ) is said to be absolutely stable if the frequency condition below is satisfied:

$\mathrm{He}\left[K^{-1}+(1+(z-1) N) G(z)-\frac{S|N|}{2}|(z-1) G(z)|^{2}\right]>0$

$\forall|z|=1$, with $S=\operatorname{diag}\left(s_{1}, \ldots, s_{p}\right)>0$ and $N=$ $\operatorname{diag}\left(n^{1}, \ldots, n^{p}\right) \in \mathbb{R}^{p \times p}$. With $P, K$ and $S$ positive definite, $N$ is indefinite with appropriate dimensions. Sharma and Singh (1981) [21] prove the Jury-Lee criterion for the diagonal MIMO case via the Lyapunov function as follows:

$V_{k}^{3}=x_{k}^{T} P x_{k}+2 \sum_{i=1}^{p} n_{i} \int_{0}^{y_{k}^{i}} \phi_{i}(\sigma) d \sigma$.

The multiplier $N$ in this case is also allowed to take negative values, which is consistent with the original Jury-Lee criterion in the frequency domain. A more recent result is presented in Haddad and Bernstein (1994) [2]. In their results, a Lyapunov function similar to (10) is constructed for the nonlinearities belonging to $\Omega(K, S)$, but the multiplier $N$ is restricted to be nonnegative definite so as to ensure the positiveness of the Lyapunov function. Note that although the Lyapunov function (10) can be shown to be positive with indefinite multiplier, the analysis in [21] does not appear to be complete (see the results in Section 2).

To summarize, for the discrete-time MIMO Lur'e system with nonlinearities that belong to $\Omega(K, \infty)$, the Lur'eLyapunov function is best expressed as in (8), which is the extension of the Tsypkin criterion [25], and with nonlinearities belonging to $\Omega(K, S)$, it is best described by the extension of the Jury-Lee criterion as in (10). It should be noted that all the existing results cited above are established for the case where the nonlinearities are diagonal (in the sense that $\phi_{i}(y)=\phi_{i}\left(y^{i}\right)$ for $i=1, \ldots, p$ ).

From the input-output stability approach, Willems and Brockett (1968) [29] provide the most general multiplier for the nonlinearity belonging to $\Omega(K, \infty)$ and $\Omega(K, S)$ in the SISO case, which is the discrete-time counterpart of ZamesFalb multiplier [30]. The possible superiority of the multiplier approach is undeniable, but it is not always easy to find the parameterization of the multiplier or to derive a criterion for a given Lur'e problem. A weaker generalization of the multiplier is also presented by Narendra and Cho (1968) [15] for the SISO case where geometrical stability criteria are given. The Lyapunov function associated with Narendra's multiplier is presented in [3], but the resulting criteria do not lead to a convex search.

As the LMI solvers for modern controls nowadays have greatly reduced the complexity of the controller analysis and synthesis, the LMI is more preferrable compared to the frequency-based approach. Most of the modern controllers are also implemented digitally, which motivates the search for stability criteria in discrete-time settings. In this note, the problem of interest is to find improved LMI-based stability criteria for the discrete-time Lur'e systems where the nonlinearities belong to $\Omega(K, S)$. A Lur'e-Lyapunov function for the system is constructed by the extension of the Jury-Lee and the Tsypkin criteria. Our main contribution is to include the extra term in the stability criterion corresponding to the slope restriction yet preserving convexity in the optimization. The result may be seen as a discrete counterpart of [16]. Although more general multipliers (such as Zames-Falb [30]) may be available for the discrete-time systems, convex searches are not known without severely restricting the subset of multipliers over which a search is carried out. In the continuous domain, [16] often gives better results than convex searches over subsets of the Zames-Falb multipliers (see [28], and subsequent discussion [1], [27]).

By employing some of the techniques from Heath and $\mathrm{Li}$ (2009) [4], the results are extended to a more general case which includes both diagonal and non-diagonal nonlinearities. The new result for the diagonal MIMO case may be applied to discrete-time anti-windup schemes for robust stability analysis and synthesis (e.g.[14]). The importance of extending the discrete-time Popov criterion to the non-diagonal case is that it can be applied to stability and robustness analysis of input-constrained model predictive control which is inherently discrete (see [5], [7], [18]).

\section{TECHNICAL RESULTS}

Consider the system (1)-(2) in negative feedback with nonlinearities that belong to $\Omega(K, S)$, where $K, S \in \mathbb{R}^{p \times p}$ are positive definite matrices. Define

$A_{a}=\left[\begin{array}{cc}A & B \\ 0 & 0\end{array}\right], \quad B_{a}=\left[\begin{array}{l}0 \\ I\end{array}\right], \quad C_{a}=\left[\begin{array}{ll}C & 0\end{array}\right]$.

Note that $\hat{x}_{k+1}=A_{a} \hat{x}_{k}+B_{a} u_{k+1}$ and $y_{k}=C_{a} \hat{x}_{k}$, where $\hat{x}_{k}=\left(x_{k}, u_{k}\right)$. We will find it useful to define

$L_{a}(z)=\left[\begin{array}{c}\left(z I-A_{a}\right)^{-1} B_{a} \\ I\end{array}\right]$.

Based on the transfer function (2), we also have the relation

$(z-1) G(z)=C(A-I)(z I-A)^{-1} B+C B$.

Throughout this note, we define $N$ to be the set of positive semi-definite multiplier $N \in \mathbb{R}^{p \times p}$. As the results derived are based on LMIs, the associated matrices are defined explicitly in the following definition.

Definition 2.1: Define $A_{-}=A_{a}-I$ and $A_{+}=A_{a}+I$. Let $K, S \in \mathbb{R}^{p \times p}$ and $P \in \mathbb{R}^{\left(n_{x}+p\right) \times\left(n_{x}+p\right)}$ be positive definite. The symmetric matrices $M_{0 P}, M_{1 a}, M_{1 b}, M_{2 a}, M_{2 b}, M_{3}, M_{4}$, and $M_{s}$ are then defined as follows

$$
\begin{aligned}
& M_{0 P}=\left[\begin{array}{cc}
A_{a}^{T} P A_{a}-P & A_{a}^{T} P B_{a} \\
B_{a}^{T} P A_{a} & B_{a}^{T} P B_{a}
\end{array}\right], \\
& M_{1 a}=\left[\begin{array}{cc}
A_{-}^{T} C_{a}^{T} S N_{1} C_{a} A_{-} & 0 \\
0 & 0
\end{array}\right], \\
& M_{1 b}=\mathrm{He}\left[\begin{array}{cc}
-N_{1} B_{a} C_{a} A_{-} & 0 \\
0 & 0
\end{array}\right], M_{2 a}=\mathrm{He}\left[\begin{array}{cc}
N_{2} B_{a} C_{a} A_{-} & 0 \\
0 & 0
\end{array}\right], \\
& M_{2 b}=\mathrm{He} \frac{1}{2}\left[\begin{array}{cc}
A_{-}^{T} C_{a}^{T} K N_{2} C_{a} A_{+} & 0 \\
0 & 0
\end{array}\right],
\end{aligned}
$$


$M_{3}=\mathrm{He}\left[\begin{array}{cc}0 & 0 \\ -N_{3} C_{a} A_{-} & 0\end{array}\right], M_{s}=\mathrm{He}\left[\begin{array}{cc}0 & 0 \\ -C_{a} A_{a} & -K^{-1}\end{array}\right]$

$M_{4}=\mathrm{He}\left[\begin{array}{cc}N_{4} B_{a}\left(C_{a} A_{-}-S^{-1} B_{a}^{T}\right) & B_{a} S^{-1} N_{4} \\ -N_{4}\left(C_{a} A_{-}-S^{-1} B_{a}^{T}\right) & -N_{4} S^{-1}\end{array}\right]$

with $N_{1}, N_{2}, N_{3}, N_{4} \in \boldsymbol{N}$.

The next lemma will be used in the main results as it is crucial in proving the general Lyapunov function in Theorem 2.1. Extra conditions need to be imposed on the nonlinearities and the multiplier $N$ so as to ensure the validity of the general case.

Lemma 2.1: Consider the nonlinearity $\phi \in \Omega(K, S)$ and $N \in N$, and let (i) $I \in N$, (ii) $S N=N S$ and $K N=N K$, (iii) there exists some continuous $\Phi_{N}: R^{p} \rightarrow R$ such that the line integral $\Phi_{N}\left(y_{k}\right)=\int_{0}^{y_{k}} \phi(\sigma)^{T} N d \sigma$ is independent of path, and (iv) $\left[N^{\frac{1}{2}}(\phi(\widehat{y})-\phi(y))\right]^{T}\left[S^{-1} N^{\frac{1}{2}}(\phi(\widehat{y})-\phi(y))-N^{\frac{1}{2}}(\widehat{y}-y)\right] \leq 0$ holds for all $\widehat{y} \neq y$. Define $\Lambda=\left(x_{k}, u_{k}, u_{k+1}\right)$. The nonlinearity $\phi$ is then bounded as follows

(a) $2 \int_{y_{k}}^{y_{k+1}} \phi(\sigma)^{T} N_{1} d \sigma \leq \Lambda^{T}\left(M_{1 a}+M_{1 b}\right) \Lambda$,

(b) $2 \int_{y_{k}}^{y_{k+1}}[K \sigma-\phi(\sigma)]^{T} N_{2} d \sigma \leq \Lambda^{T}\left(M_{2 a}+M_{2 b}\right) \Lambda$,

(c) $2 \int_{y_{k}}^{y_{k+1}} \phi(\sigma)^{T} N_{3} d \sigma \leq \Lambda^{T}\left(M_{3}\right) \Lambda$,

with $N_{1}, N_{2}, N_{3} \in N$, and $M_{0 P}, M_{1 a}, M_{1 b}, M_{2 a}, M_{2 b}$ and $M_{3}$ defined as in Definition 2.1.

Proof: See Appendix.

The following definition is required for the results.

Definition 2.2: Based on Definition 2.1, define $M_{p}$ and $M_{q}$ as follows

$M_{p}=M_{1 a}+M_{1 b}+M_{2 a}+M_{2 b}+M_{3}+M_{4}+M_{s}$,

$M_{q}=M_{1 a}+M_{1 b}+M_{2 a}+M_{3}+M_{4}+M_{s}$.

Now we can state our main theorem which gives a new LMI condition (21) for testing the stability of discrete-time Lur'e systems.

Theorem 2.1: Consider the system (1)-(2) with the nonlinearity $\phi \in \Omega(K, S)$ satisfying (4) and (5) where $K, S \in \mathbb{R}^{p \times p}$ are positive definite. The feedback interconnection is assumed to be well-posed. Assume conditions (i) to (iv) of Lemma 2.1 are satisfied, and (v) $\phi\left(y_{k}\right)^{T} N^{\frac{1}{2}}\left(K^{-1} N^{\frac{1}{2}} \phi\left(y_{k}\right)-N^{\frac{1}{2}} y_{k}\right) \leq 0$ holds for all $y_{k}$. Then the system is absolutely stable if there exist a positive definite matrix $P$ and $N_{1}, N_{2}, N_{3}, N_{4} \in N$ such that

$M_{0 P}+M_{p}<0$

with $M_{0 P}$ and $M_{p}$ defined as in Definitions 2.1 and 2.2.

Proof: See Appendix.

Remark 2.1: The contribution of Theorem 2.1 is the term $M_{4}$ in the LMI (21) via (15), which corresponds to the slope restriction and is introduced via the S-procedure.

The condition (i) ensures that there exists some continuous $\Phi: \mathbb{R}^{p \times p}$ such that $\nabla \Phi=\phi(\sigma)$, and this needs to be emphasized mainly for the non-diagonal MIMO case to show that the Jacobian matrix of the nonlinearities is always symmetric [20], [4]. As for the diagonal nonlinearities, the conditions (i) to (v) are naturally satisfied as there are no cross-terms between the inputs and the outputs of the nonlinearities. The next corollary shows that the LMI in Theorem 2.1 can also be simplified to another smaller LMI as they share the same frequency condition.

Corollary 2.1: Under the conditions of Theorem 2.1, let $\hat{G}(z)$ be defined as follows

$$
\begin{gathered}
\hat{G}(z)=K^{-1}+|z-1|^{2} S^{-1} N_{4}-\frac{1}{2}|z-1|^{2} G(z)^{*} N_{1} S G(z)+ \\
{\left[I+(z-1)\left(N_{1}-N_{2}\right)+\left(1-z^{-1}\right) N_{3}+|z-1|^{2} N_{4}\right] G(z) .}
\end{gathered}
$$

With $M_{0 P}, M_{p}$ and $M_{q}$ defined as in Definitions 2.1 and 2.2, the following statements are equivalent:

(a) there exists some $P_{a}>0$ such that $M_{0 P_{a}}+M_{p}<0$,

(b) there exists some $P_{b}>0$ such that $M_{0 P_{b}}+M_{q}<0$,

(c) $A_{a}$ is Schur and $\mathrm{He}[\hat{G}(z)]>0 \quad \forall|z|=1$.

Proof: See Appendix.

Remark 2.2: The frequency-domain condition (c) in Corollary 2.1 may also be expressed in terms of the integral quadratic constraint (IQC) [8] as follows:

$\left[\begin{array}{c}-G(z) \\ I\end{array}\right]^{*}\left[\begin{array}{cc}Q_{1}(z) & * \\ Q_{2}(z) & Q_{3}(z)\end{array}\right]\left[\begin{array}{c}-G(z) \\ I\end{array}\right] \leq-\epsilon I$

for all $|z|=1$, where $Q_{1}(z)=|z-1|^{2} G(z)^{*} N_{1} S G(z), Q_{2}(z)=$ $-\left[I+(z-1)\left(N_{1}-N_{2}\right)+\left(1-z^{-1}\right) N_{3}+|z-1|^{2} N_{4}\right], Q_{3}(z)=$ $2 K^{-1}-2|z-1|^{2} S^{-1} N_{4}$ and $\epsilon>0$.

The new criterion is applicable to the SISO, diagonal MIMO and also general cases. As for the case where the nonlinearity is diagonal, its scaled version is introduced in the following definition and corollary (see [17] for an example of the scaled Tsypkin criterion).

Definition 2.3: Following Definition 2.1, the symmetric matrices $\tilde{M}_{1 a}, \tilde{M}_{1 b}, \tilde{M}_{2 a}, \tilde{M}_{3}, \tilde{M}_{4}$ and $\tilde{M}_{s}$ are defined as follows

$$
\begin{aligned}
\tilde{M}_{1 a} & =\left[\begin{array}{cc}
A_{-}^{T} C_{a}^{T} \bar{N}_{1} C_{a} A_{-} & 0 \\
0 & 0
\end{array}\right], \\
\tilde{M}_{1 b} & =\operatorname{He}\left[\begin{array}{cr}
-\bar{N}_{1} B_{a} C_{a} A_{-} & 0 \\
0 & 0
\end{array}\right], \tilde{M}_{2 a}=\operatorname{He}\left[\begin{array}{cc}
\bar{N}_{2} B_{a} C_{a} A_{-} & 0 \\
0 & 0
\end{array}\right], \\
\tilde{M}_{3} & =\operatorname{He}\left[\begin{array}{cc}
0 & 0 \\
-\bar{N}_{3} C_{a} A_{-} & 0
\end{array}\right], \tilde{M}_{s}=\operatorname{He}\left[\begin{array}{cc}
0 & 0 \\
-\Sigma C_{a} A_{a} & -\Sigma K^{-1}
\end{array}\right], \\
\tilde{M}_{4} & =\operatorname{He}\left[\begin{array}{cc}
\bar{N}_{4} B_{a} C_{a} A_{-}-B_{a} \bar{N}_{4} B_{a}^{T} & B_{a} \bar{N}_{4} \\
-\bar{N}_{4} C_{a} A_{-}+\bar{N}_{4} B_{a}^{T} & -\bar{N}_{4}
\end{array}\right],
\end{aligned}
$$

where $\Pi=\operatorname{diag}\left(\alpha_{1}, \ldots, \alpha_{p}\right) \in \mathbb{R}^{p \times p}$ with $\Pi>0, \bar{N}_{i}=$ $\Pi^{T} N_{i} \Pi \geq 0$ for $i=1, \ldots, 4$, and $\Sigma=\Pi^{T} \Pi>0$.

Corollary 2.2: (A new scaled criterion.) Let the nonlinearities (3), the sector and slope matrices $K$ and $S$, and the multipliers $N_{1}, N_{2}, N_{3}$ and $N_{4}$ be set to diagonal with appropriate dimensions. Following the results of Corollary 2.1, let $\hat{G}_{2}(z)$ be defined as follows

$$
\begin{aligned}
\hat{G}_{2}(z)= & K^{-1}+|z-1|^{2} S^{-1} N_{4}+\left[I+(z-1)\left(N_{1}-N_{2}\right)\right. \\
& \left.+\left(1-z^{-1}\right) N_{3}+|z-1|^{2} N_{4}\right] \Pi G(z) \Pi^{-1} \\
& -\frac{1}{2}|z-1|^{2} \Pi^{-T} G^{*}(z) \Pi^{T} S N_{1} \Pi G(z) \Pi^{-1}
\end{aligned}
$$


For fixed values of $S$ and $K$, the system is absolutely stable if He $\hat{G}_{2}(z)>0$ for all $|z|=1$, or equivalently, if there exist $P>$ $0, N_{i} \geq 0$ for $i=1, \ldots, 4$, and $\Sigma>0$ such that $M_{0 P}+\tilde{M}_{1 a}+$ $\tilde{M}_{1 b}+\tilde{M}_{2 a}+\tilde{M}_{3}+\tilde{M}_{4}+\tilde{M}_{s}<0$ with $\tilde{M}_{1 a}, \tilde{M}_{1 b}, \tilde{M}_{2 a}, \tilde{M}_{3}, \tilde{M}_{4}$ and $\tilde{M}_{s}$ defined as in Definition 2.3.

Proof: See Appendix.

\section{COMPARISONS WITH EXISTING CRITERIA}

The frequency-domain criterion (22) and its correponding Lyapunov function (27) are constructed with four positive semi-definite multipliers: $N_{1}, N_{2}, N_{3}$, and $N_{4}$. If the nonlinearities and the multipliers are set to diagonal with approproate dimensions, the new result is then comparable with the existing discrete-time Popov-like criteria. It generalizes them as follows:

- when $N_{1}=N_{2}=N_{3}=N_{4}=0$, the criterion reduces to the circle criterion [24],

- when $N_{2}=N_{3}=N_{4}=0$, the criterion reduces to that of Haddad and Bernstein [2],

- when $N_{2}=N_{4}=0$, the criterion reduces to that of Soliman and Kwoh [22],

- when $N_{1}=N_{2}=N_{4}=0$, the criterion reduces to that of Kapila and Haddad [12], and

- when $N_{1}=N_{4}=0$, the criterion reduces to that of Park and Kim [17].

From the comparisons made above, the term associated with $N_{4}$ appears to have no counterpart in the literature. It plays an important role in making the proposed stability criterion less conservative than the existing ones.

\section{APPLICATIONS}

\section{A. Application to SISO nonlinearities}

Systems with monotonic, slope-bounded nonlinearities arise in a variety of control problems. The most common examples are systems having saturations in the actuators.

Example 1.

$G_{1}(z)=\frac{-0.5 z+0.1}{\left(z^{2}-z+0.89\right)(z+0.1)}$

Consider the example for the SISO case from [17] where a stable, discrete-time plant (24) is subject to a control saturation. To identify the new approach, the maximum upper slope bound at which the system is stable is computed for each criterion (see Table I). In the case where the saturation is replaced by a unit gain, the Nyquist criterion provides the maximum slope bound, and it can be seen that the new approach gives the best result for this example.

\section{B. Application to non-diagonal nonlinearities}

If the nonlinearity $\phi$ is represented by a quadratic program (QP) as follows:

$\phi(y)=\underset{v}{\arg \min } \frac{1}{2} v^{T} H v-v^{T} y \quad$ s.t. $\quad L v \leq b \quad$ with $\quad b \geq 0$

with fixed Hessian matrix $H>0$ and fixed $L$ and $b$, the KKT conditions can be used to show that the nonlinearity satisfies the sector bound condition $\phi(y)^{T}(H \phi(y)-y) \leq 0$
Table I

EXAMPLE 1

\begin{tabular}{|l|l|c|c|c|c|}
\hline Criterion & $\begin{array}{l}\text { Maximum } \\
\text { slope }\end{array}$ & $n_{1}$ & $n_{2}$ & $n_{3}$ & $n_{4}$ \\
\hline Circle Criterion[24] & 1.0273 & N/A & N/A & N/A & N/A \\
\hline $\begin{array}{l}\text { Kapila \& } \\
\text { Haddad[12] }\end{array}$ & 1.0273 & N/A & N/A & 0 & N/A \\
\hline Park \& Kim [17] & 1.7252 & N/A & 0.75638 & 0 & N/A \\
\hline $\begin{array}{l}\text { Soliman \& Kwoh } \\
{[22]}\end{array}$ & 1.0273 & 0 & N/A & 0 & N/A \\
\hline $\begin{array}{l}\text { Haddad \& Bernstein } \\
\text { [2] }\end{array}$ & 1.0273 & 0 & N/A & N/A & N/A \\
\hline Theorem 2.1 & 2.4474 & 0 & 6.5999 & 0 & 4.8657 \\
\hline Nyquist Criterion & 2.4475 & N/A & N/A & N/A & N/A \\
\hline
\end{tabular}

[7] and the monotonic and slope-bound condition $[\phi(y)-$ $\phi(\hat{y})]^{T}[H[\phi(y)-\phi(\hat{y})]-(y-\hat{y})] \leq 0$ [18] [6] [13]. Such conditions arise naturally in some input-constrained MPC [7][13].

Therefore, in order to apply the new criteria to such a QP, we assume, in general, no additional structure on $\phi$ beyond the conditions of Theorem 2.1. Choose $N_{1}, N_{2}, N_{3}$ and $N_{4}$ to be $n_{1} I, n_{2} I, n_{3} I$ and $n_{4} I$ respectively so that the conditions (i)-(v) (in Lemma 2.1 and Theorem 2.1) are satisfied. Then set $K^{-1}=S^{-1}=H$.

The next part example shows a non-diagonal MIMO case where the nonlinearities are unstructured. A stable, strictly proper plant $G_{2}(z)$ is in negative feedback with a controller that is expressed by a QP with a positive definite Hessian matrix $H_{2}$. The plant's output is multiplied by a positive constant gain $\mathrm{k}$, and the maximum stable gain for the new criterion is compared with the generalized circle criterion [7].

Example 2. The 2-input-2-output plant and the Hessian matrix are given by

$$
\begin{aligned}
G_{2}(z) & =\frac{0.1 z-0.04}{(z-0.9)(z-0.1)(z-0.8)}\left[\begin{array}{ll}
5 & 2 \\
3 & 4
\end{array}\right] \text { and } \\
H_{2} & =\left[\begin{array}{ll}
6 & 0 \\
0 & 3
\end{array}\right]
\end{aligned}
$$

respectively. The generalized circle criterion will give a maximum gain of $k=1.1877$. Application of the new criterion will give a higher maximum gain, which is $k=3.1069$ with $n_{1}=22.06$ and $n_{4}=7.23$.

Remark 4.1: In this case, the conditions of [18][13] give $k=2.2070$. However, there is no guarantee that the proposed method beats [18][13] and the combination is the subject of current research.

\section{DISCUSSIONS AND CONCLUSIONS}

As can be seen from the examples, the introduction of the multiplier $N_{4}$ in the new criteria can make an improvement even in the SISO case. The additional term associated with $N_{4}$ in the LMI accurately describes the slope condition of the nonlinearity. The performance of the new criterion for the non-diagonal MIMO example is also shown to be better than the generalized circle criterion. In conclusion, for the nonlinearity which is pre-specified to be monotonic, sectorand slope-restricted, the new LMI-based criteria provide better 
results than the other existing LMI-based Popov-like criteria in the literature. Although they might be weaker than multiplierbased criteria, they are more computationally tractable.

\section{ACKNOWLEDGMENT}

The authors would like to thank Dr Joaquin Carrasco for some useful suggestions and discussion, and the anonymous reviewers for the constructive comments.

\section{APPENDIX}

\section{A. Proof of Lemma 2.1(a)}

Since $S>0$, we have the inequality

$$
\begin{aligned}
& {\left[S^{-1} N^{\frac{1}{2}}\left(\phi(\sigma)-\phi(y)-N^{\frac{1}{2}}(\sigma-y)\right]^{T} S .\right.} \\
& \quad\left[S^{-1} N^{\frac{1}{2}}(\phi(\sigma)-\phi(y))-N^{\frac{1}{2}}(\sigma-y)\right] \geq 0 .
\end{aligned}
$$

Combining this with condition (iv) gives

$\left[N^{\frac{1}{2}}(\sigma-y)\right]^{T}\left[N^{\frac{1}{2}}(\phi(\sigma)-\phi(y))-S N^{\frac{1}{2}}(\sigma-y)\right] \leq 0$. It follows immediately that

$\left[N^{\frac{1}{2}}(\sigma-y)\right]^{T}\left[N^{\frac{1}{2}}(\phi(\sigma)-\phi(y))\right] \leq\left[N^{\frac{1}{2}}(\sigma-y)\right]^{T} S\left[N^{\frac{1}{2}}(\sigma-y)\right]$, or equivalently, $[\sigma-y]^{T} N[\phi(\sigma)-\phi(y)] \leq[\sigma-y]^{T} S N[\sigma-y]$.

This proves that $[\phi(\sigma)-\phi(y)]^{T} N(\sigma-y) \leq[\sigma-y]^{T} S N[\sigma-y]$. Let $y=y_{k}$ and parameterize $\sigma=y_{k}+\lambda\left(y_{k+1}-y_{k}\right)$, with $\lambda \in[0,1]$ and $d \sigma=\left(y_{k+1}-y_{k}\right) d \lambda$, we can write

$$
\begin{aligned}
& {\left[\phi\left(y_{k}+\lambda\left(y_{k+1}-y_{k}\right)\right)-\phi\left(y_{k}\right)\right]^{T} N \lambda\left(y_{k+1}-y_{k}\right)} \\
& \quad \leq\left[y_{k+1}-y_{k}\right]^{T} \lambda^{2} S N\left[y_{k+1}-y_{k}\right] .
\end{aligned}
$$

Since $\lambda \geq 0$, then

$\left[\phi\left(y_{k}+\lambda\left(y_{k+1}-y_{k}\right)\right)-\phi\left(y_{k}\right)\right]^{T} N\left(y_{k+1}-y_{k}\right) \leq$ $\left[y_{k+1}-y_{k}\right]^{T} \lambda S N\left[y_{k+1}-y_{k}\right]$. Integrating this gives

$$
\begin{gathered}
\int_{0}^{1}\left[\phi\left(y_{k}+\lambda\left(y_{k+1}-y_{k}\right)\right)-\phi\left(y_{k}\right)\right]^{T} N\left(y_{k+1}-y_{k}\right) d \lambda \\
\leq \int_{0}^{1}\left[y_{k+1}-y_{k}\right]^{T} \lambda S N\left[y_{k+1}-y_{k}\right] d \lambda
\end{gathered}
$$

and hence $\int_{y_{k}}^{y_{k+1}}\left[\phi(\sigma)-\phi\left(y_{k}\right)\right]^{T} N d \sigma \leq \int_{y_{k}}^{y_{k+1}}\left(\sigma-y_{k}\right) S N d \sigma$. This is sufficient to ensure

$$
\begin{aligned}
\int_{y_{k}}^{y_{k+1}}\left[\phi(\sigma)-\phi\left(y_{k}\right)\right]^{T} N d \sigma & \leq \int_{y_{k}}^{y_{k+1}}\left(\sigma-y_{k}\right) S N d \sigma \\
& =\frac{1}{2}\left[y_{k+1}-y_{k}\right]^{T} S N\left[y_{k+1}-y_{k}\right] .
\end{aligned}
$$

Hence

$$
\begin{aligned}
& 2 \int_{y_{k}}^{y_{k+1}} \phi(\sigma)^{T} N_{1} d \sigma \\
& \quad \leq 2 \phi\left(y_{k}\right)^{T} N_{1}\left(y_{k+1}-y_{k}\right)+\left[y_{k+1}-y_{k}\right]^{T} S N_{1}\left[y_{k+1}-y_{k}\right] \\
& \quad=\text { Right-hand side of (16) }
\end{aligned}
$$

\section{B. Proof of Lemma 2.1(b)}

We have $\left[N^{\frac{1}{2}}(\phi(\sigma)-\phi(y))\right]^{T} S^{-1}\left[N^{\frac{1}{2}}(\phi(\sigma)-\phi(y))\right] \geq 0$.

Combining this with condition (iv) gives

$\left[N^{\frac{1}{2}}(\phi(\sigma)-\phi(y))\right]^{T}\left[N^{\frac{1}{2}}(\sigma-y)\right] \geq 0$, or equivalently

$[\phi(\sigma)-\phi(y)]^{T} N[\sigma-y] \geq 0$, and hence

$\phi(\sigma)^{T} N[\sigma-y] \geq \phi(y)^{T} N[\sigma-y]$. Let $y=y_{k}$ and parameterize $\sigma=y_{k}+\lambda\left(y_{k+1}-y_{k}\right)$, with $\lambda \in[0,1]$ and $d \sigma=\left(y_{k+1}-y_{k}\right) d \lambda$. We can write

$\phi\left(y_{k}+\lambda\left(y_{k+1}-y_{k}\right)\right)^{T} N \lambda\left(y_{k+1}-y_{k}\right) \geq \phi\left(y_{k}\right)^{T} N \lambda\left(y_{k+1}-y_{k}\right)$.

Since $\lambda \geq 0$, then

$\phi\left(y_{k}+\lambda\left(y_{k+1}-y_{k}\right)\right)^{T} N\left(y_{k+1}-y_{k}\right) \geq \phi\left(y_{k}\right)^{T} N\left(y_{k+1}-y_{k}\right)$.

Integrating this gives

$$
\begin{array}{r}
\int_{0}^{1} \phi\left(y_{k}+\lambda\left(y_{k+1}-y_{k}\right)\right)^{T} N\left(y_{k+1}-y_{k}\right) d \lambda \geq \\
\int_{0}^{1} \phi\left(y_{k}\right)^{T} N\left(y_{k+1}-y_{k}\right) d \lambda
\end{array}
$$

and hence

$\int_{y_{k}}^{y_{k+1}} \phi(\sigma)^{T} N d \sigma \geq \int_{y_{k}}^{y_{k+1}} \phi\left(y_{k}\right)^{T} N d \sigma$.

Therefore $\int_{y_{k}}^{y_{k+1}} \phi(\sigma)^{T} N d \sigma \geq \phi\left(y_{k}\right)^{T} N\left(y_{k+1}-y_{k}\right)$. Hence

$$
\begin{aligned}
-2 \int_{y_{k}}^{y_{k+1}} \phi(\sigma)^{T} N_{2} d \sigma & \leq-2 \phi\left(y_{k}\right)^{T} N_{2}\left(y_{k+1}-y_{k}\right) \\
& =\Lambda^{T}\left(M_{2 a}\right) \Lambda .
\end{aligned}
$$

The inequality (17) then holds since

$2 \int_{y_{k}}^{y_{k+1}}[K \sigma]^{T} N_{2} d \sigma=\left[\sigma^{T} K N_{2} \sigma\right]_{y_{k}}^{y_{k+1}}=\Lambda^{T}\left(M_{2 b}\right) \Lambda$.

\section{Proof of Lemma 2.1(c)}

Since we have $\sigma=y_{k}+\lambda\left(y_{k+1}-y_{k}\right)$ with $\lambda \in[0,1]$ in (26), it follows immediately that

$$
\begin{aligned}
& \int_{y_{k}}^{y_{k+1}} \phi(\sigma)^{T} N d \sigma \\
& \quad \leq \int_{y_{k}}^{y_{k+1}} \phi\left(y_{k+1}\right)^{T} N d \sigma=\phi\left(y_{k+1}\right)^{T} N\left(y_{k+1}-y_{k}\right) .
\end{aligned}
$$

Hence the result.

\section{Proof of Theorem 2.1}

Let the corresponding Lur'e-Lyapunov function of the system be as follows:

$$
\begin{aligned}
V\left(\hat{x}_{k}\right)= & \hat{x}_{k}^{T} P \hat{x}_{k}+2 \int_{0}^{y_{k}} \phi(\sigma)^{T}\left(N_{1}+N_{3}\right) d \sigma \\
& +2 \int_{0}^{y_{k}}[K \sigma-\phi(\sigma)]^{T} N_{2} d \sigma
\end{aligned}
$$

with $N_{1}, N_{2}, N_{3} \in \boldsymbol{N}$. In order to prove the absolute stability of the system using the Lyapunov stability theory, we need to show that the function (27) is radially unbounded by $\hat{x}_{k}$ and its difference equation $\Delta V=V_{k+1}-V_{k}$ is negative definite for all nonzero $\hat{x}_{k}$. Inequality (v) ensures that $\phi\left(y_{k}\right)^{T}\left(N_{1}+N_{3}\right) y_{k} \geq 0$ for all $y_{k}$, and hence $\int_{0}^{y_{k}} \phi(\sigma)^{T}\left(N_{1}+N_{3}\right) d \sigma \geq 0$. Since $K>0$, we have the inequality $\left(K^{-1} N^{\frac{1}{2}} \phi\left(y_{k}\right)-N^{\frac{1}{2}} y_{k}\right)^{T} K\left(K^{-1} N^{\frac{1}{2}} \phi\left(y_{k}\right)-N^{\frac{1}{2}} y_{k}\right) \geq 0$. This, with (v), gives $y_{k}^{T} N^{\frac{1}{2}} K N^{\frac{1}{2}} y_{k}-\phi\left(y_{k}\right)^{T} N y_{k} \geq 0$, or, equivalently, $\left[K y_{k}-\phi\left(y_{k}\right)\right]^{T} N y_{k} \geq 0$. This is sufficient to ensure that $\int_{0}^{y_{k}}(K \sigma-\phi(\sigma))^{T} N_{2} d \sigma \geq 0$. Therefore we can say that $V_{k} \geq \hat{x}_{k}^{T} P \hat{x}_{k}$ for some $P>0$ (i.e. the Lyapunov function is positive definite for all $\hat{x}_{k}$ and is radially unbounded by $\hat{x}_{k}$ ). 
Now, we need to prove that $\Delta V \leq-\epsilon\left|\hat{x}_{k}\right|^{2}$ for some $\epsilon>0$. Subtracting $V_{k}$ from $V_{k+1}$, gives

$$
\begin{aligned}
\Delta V=\quad & \hat{x}_{k+1}^{T} P \hat{x}_{k+1}-\hat{x}_{k}^{T} P \hat{x}_{k}+2 \int_{y_{k}}^{y_{k+1}} \phi(\sigma)^{T}\left(N_{1}+N_{3}\right) d \sigma \\
& +2 \int_{y_{k}}^{y_{k+1}}[K \sigma-\phi(\sigma)]^{T} N_{2} d \sigma .
\end{aligned}
$$

Then we have $\hat{x}_{k+1}^{T} P \hat{x}_{k+1}-\hat{x}_{k}^{T} P \hat{x}_{k}=\Lambda^{T}\left(M_{0 P}\right) \Lambda$ and following Lemma 2.1, we will get $\Delta V \leq$ $\Lambda^{T}\left(M_{0 P}+M_{1 a}+M_{1 b}+M_{2 a}+M_{2 b}+M_{3}\right) \Lambda$. But the sector bound of $\phi$ says $\Lambda^{T}\left(M_{s}\right) \Lambda \geq 0$, and the slope bound (iv) gives $\Lambda^{T}\left(M_{4}\right) \Lambda \geq 0$. Application of the $\mathrm{S}$-procedure gives $\Delta V \leq$

$\Lambda^{T}\left(M_{0 P}+M_{1 a}+M_{1 b}+M_{2 a}+M_{2 b}+M_{3}+M_{4}+M_{s}\right) \Lambda$.

It follows that $\Delta V$ is negative definite for all nonzero $\Lambda$, provided (21) holds, and hence the system is absolutely stable.

\section{E. Proof of Corollary 2.1}

Invoking the discrete KYP lemma [19], statement (a) which is similar to the LMI in (21) can be written as

$L_{a}(z)^{*}\left(M_{p}\right) L_{a}(z)<0 \quad \forall|z|=1$

with $L_{a}(z)$ defined in (12). This is also equivalent to $-\mathrm{He}[\hat{G}(z)]<0$ for all $|z|=1$. It follows immediately that the statement (a) is equivalent to statement (c), but we also have $L_{a}(z)^{*}\left(M_{2 b}\right) L_{a}(z)=0$ for all $|z|=1$. Therefore, the frequency domain inequality (28) is also equivalent to $L_{a}(z)^{*}\left(M_{q}\right) L_{a}(z)<0$ for all $|z|=1$. Invoking the discrete KYP Lemma again leads to statement (b). Hence statements (a) and (b) are also equivalent. Note also that the top left entries of both $M_{q}$ and $M_{p}$ are positive semidefinite due to the integrals (a)-(c) in Lemma 2.1, so it is easy to prove that $A_{a}$ is Schur stable.

\section{F. Proof of Corollary 2.2}

Pre- and post-multiplying $\mathrm{He} \hat{G}_{2}(z)>0$ by $\Pi^{T}$ and $\Pi$ respectively gives

$L_{a}(z)^{*}\left(\tilde{M}_{1 a}+\tilde{M}_{1 b}+\tilde{M}_{2 a}+\tilde{M}_{3}+\tilde{M}_{4}+\tilde{M}_{s}\right) L_{a}(z)<0$.

By using the discrete KYP lemma, we will get the LMI. And since $A$ is Schur stable, there exists a positive definite $P$.

\section{REFERENCES}

[1] J. Carrasco, W.P. Heath, G. Li, and A. Lanzon. Comments on 'On the existence of stable, causal multipliers for systems with slope-restricted nonlinearities'. IEEE Transactions On Automatic Control (to appear, available online), 2012.

[2] W.M. Haddad and D.S. Bernstein. Explicit construction of quadratic Lyapunov functions for the small gain, positivity, circle and Popov theorems and their application to robust stability. Part II: Dicrete-time theory. International Journal of Robust and Nonlinear Control, 4:249265, 1994

[3] W.M. Haddad and V. Kapila. Discrete-time extensions of mixed- $\mu$ bounds to monotonic and odd-monotonic nonlinearities. International Journal Control, 61:423-441, 1995.

[4] W.P. Heath and G. Li. Lyapunov functions for the multivariable Popov criterion with indefinite multipliers. Automatica, 45:2977-2981, 2009.
[5] W.P. Heath, G. Li, A.G. Wills, and B. Lennox. The robustness of input constrained model predictive control to infinity-norm bound model uncertainty. 5th IFAC Symposium on Robust Control Design, Toulouse, France, 2006.

[6] W.P. Heath and A.G. Wills. Zames-Falb multipliers for quadratic programming. IEEE Trans. on Automatic Control, 52(10):1948-1951, 2007.

[7] W.P. Heath, A.G. Wills, and J.A.G. Akkermans. A sufficient condition for the stability of optimizing controllers with saturating actuators. International Journal of Robust and Nonlinear Control, 15:515-529, 2005.

[8] U. Jönsson. Lecture Notes on Integral Quadratic Constraints. Department of Mathematics, KTH, Stockholme, ISBN 1401-2294, available at http://www.math.kth.se/ uj/, 2001.

[9] E.I. Jury and B.W. Lee. On the stability of a certain class of nonlinear sampled-data systems.

[10] E.I. Jury and B.W. Lee. On the stability of nonlinear sampled-data systems.

[11] E.I. Jury and B.W. Lee. The absolute stability of systems with many nonlinearities. Automation and Remote Control, 26:943-961, 1965.

[12] V. Kapila and W.M. Haddad. A multivariable extension of the Tsypkin criterion using a Lyapunov-function approach. IEEE Transactions on Automatic Control, 41:149-152, 1996.

[13] G. Li, W.P. Heath, and B. Lennox. Concise stability conditions for systems with static nonlinear feedback expressed by a quadratic program. IET Control Theory and Applications, 2:554-563, 2008.

[14] M. Massimetti, L. Zacearian, T. Hu, and A. R. Teel. LMI-based linear anti-windup for discrete time linear control systems. In Proceedings of the IEEE Conference on Decision and Control, pages 6173-6178, 2006.

[15] K.S. Narendra and Y.S. Cho. Stability analysis of nonlinear and timevarying systems. SIAM Journal of Control, 6:625-646, 1968.

[16] P. Park. Stability criteria of sector- and slope-restricted Lur'e systems. IEEE Transactions on Automatic Control, 47:308-313, 2002.

[17] P. Park and S.W. Kim. A revisited Tsypkin criterion for discrete-time nonlinear Lur'e systems with monotonic sector-restrictions. Automatica, 34:1417-1420, 1998.

[18] J.A. Primbs. The analysis of optimization based controllers. Automatica, 37:933-938, 2001.

[19] A. Rantzer. On the Kalman-Yakubovich-Popov lemma. Systems and Control Letters, 28:7-10, 1996.

[20] M.G. Safonov and V.V. Kulkarni. Zames-Falb multipliers for MIMO nonlinearities. Int. J. Robust Nonlinear Control, 10:1025-1038, 2000.

[21] T.N. Sharma and Vimal Singh. On the absolute stability of multivariable discrete-time nonlinear systems. IEEE Transactions on Circuits and Systems, 26:585-586, 1981.

[22] J.I. Soliman and H.Kwoh. Absolute stability of a class of nonlinear sampled-data systems. Proceedings of the IEEE, 116:145-148, 1969.

[23] G.P. Szegö and J.B. Pearson. On the absolute stability of nonlinear sampled-data systems : The indirect case. IEEE Transactions on Automatic Control, AC-9:160-163, 1964.

[24] Y.Z. Tsypkin. On the stability in the large of nonlinear sampled-data systems. Dokl. Akad. Nauk SSSR, 145:52-55, 1962.

[25] Y.Z. Tsypkin. A criterion for absolute stability of automatic pulse systems with monotonic characteristics of the nonlinear element. Dokl. Akad. Nauk., 155:1029-Ü1932, 1964.

[26] Y.Z. Tsypkin. Frequency criteria for the absolute stability of nonlinear sampled-data systems. Automatika i Telemekhanika, 25:281-290, 1964.

[27] M. Turner, M. Kerr, and I. Postlethwaite. Authors reply to 'Comments on 'On the existence of stable, causal multipliers for systems with sloperestricted nonlinearities". IEEE Transactions on Automatic Control (to appear, available online), 2012.

[28] M.C. Turner, M. Kerr, and I. Postlethwaite. On the existence of stable, causal multipliers for systems with slope-restricted nonlinearities. IEEE Transactions on Automatic Control, 54:2697-2702, 2009.

[29] J.C. Willems and R.W. Brockett. Some new arrangement inequalities having application in stability analysis. IEEE Transactions On Automatic Control, 13:539-549, 1968

[30] G. Zames and P.L. Falb. Stability conditions for systems with monotone and slope-resctricted nonlinearities. SIAM J. Control, 6:89Ű-108, 1968. 